\title{
Entropy Budget of the Atmosphere
}

\author{
José Pinto Peixoto, ${ }^{1}$ Abraham H. Oort, ${ }^{2}$ Mário de Almeida, ${ }^{3,4}$ and António Tomé ${ }^{1}$
}

\begin{abstract}
The balance equations for the entropy in the atmosphere are presented and discussed. Using observed energy fluxes and atmospheric temperatures, we present estimates of the various terms in the global mean entropy budget. The largest boundary fluxes of entropy are associated with the emission of longwave radiation. The fluxes of entropy associated with turbulent and molecular diffusion are found to be much smaller. On the planetary scale the mean outgoing flux of entropy at the top of the atmosphere is found to be about 22 times larger than the mean incoming flux of entropy through solar radiation. The rates of entropy production and destruction by the various irreversible processes that occur in the atmosphere are also computed. The entropy production terms involved in the release of latent heat and the absorption of solar radiation are, by far, the largest sources of entropy for the atmosphere, whereas the production of entropy associated with the absorption of longwave radiation is an order of magnitude smaller. The destruction is mainly accomplished through cooling by outgoing longwave radiation. The contributions of the sensible heat fluxes and friction are relatively small. Regional contributions to the total entropy generation in the atmosphere are studied by considering an equatorial region bounded by two latitudinal walls at $15^{\circ} \mathrm{N}$ and $15^{\circ} \mathrm{S}$ and a polar region poleward of $70^{\circ} \mathrm{N}$. The rates of entropy generation by the various diabatic processes are highest in the equatorial region; part of the generated entropy is exported to higher latitudes.
\end{abstract}

\section{INTRODUCTION}

Most natural phenomena occurring in the climate system are characterized by great irreversibility. For example, the turbulent motion in the planetary boundary layer does not spontaneously develop into the large-scale organized flow of the general circulation; a cloud cannot be reconstituted from the same water it lost previously through precipitation; rivers do not flow backward from the sea to their headwaters; and ocean water does not decompose spontaneously into oxygen and hydrogen.

We will analyze the entropy budget of the atmosphere since it is important for the study of the thermodynamical behavior of the climatic system and, in particular, for atmospheric phenomena. The second law for an isolated system,

$$
d S / d t \geq 0
$$

implies that energy can only change from a higher to a lower level of availability, that is, it can only change from a more to a less usable form of energy. The second law implies the existence of a function $S$, entropy, that for an isolated system increases monotonically until it reaches its maximum value at the state of thermodynamic equilibrium. An increase in entropy of a system means a decrease in available energy and an evolution toward a state of greater disorder.

The second law can also be extended to open systems that exchange energy and matter with their surroundings. In this case, following Prigogine [1962], the total variation, $d S$, of entropy for a system is the sum of two components (see

\footnotetext{
${ }^{1}$ Geophysical Institute, University of Lisbon, Portugal.

${ }^{2}$ Geophysical Fluid Dynamics Laboratory, NOAA, Princeton University, Princeton, New Jersey.

${ }^{3}$ National Institute of Geophysics and Meteorology, Lisbon, Portugal.

${ }^{4}$ European Centre for Medium-Range Weather Forecasts, Reading, England.

Copyright 1991 by the American Geophysical Union.

Paper number 91JD00721.

0148-0227/91/91JD-00721\$05.00
}

Figure 1): the transfer, $d_{e} S$, of entropy across the boundaries of the open system and the entropy produced, $d_{i} S$, within the system. According to the second law the generation inside the system is always nonnegative, $d_{i} S \geq 0$. Thus

$$
d S=d_{e} S+d_{i} S \quad d_{i} S \geq 0
$$

where $d_{i} S=0$ for reversible processes and $d_{i} S>0$ for irreversible internal processes and where $d_{e} S$ could be positive, negative, or zero. Expression (1) can be written in terms of the rates of change:

$$
\frac{d S}{d t}=\frac{d_{e} S}{d t}+\frac{d_{i} S}{d t} \quad d_{i} S / d t \geq 0 .
$$

Using this formulation, the basic differences between reversible and irreversible processes become clearer. There are numerous irreversible processes occurring in the climate system that lead to an increase of entropy. Among these are the interaction between radiation and matter, some of the phase transitions of water substance, frictional dissipation, and molecular diffusion.

Given the continuous increase in entropy that must have taken place during the course of the Earth's history, how then can we explain why a global threshold value has not been reached? How can we explain the high level of organization in certain atmospheric processes characterizing the weather and the fact that these processes can evolve in an orderly way that at first sight would seem to lead to a decrease of entropy? For example, the zonal wind systems in the atmospheric general circulation are well-defined and highly organized; millions of tons of water evaporate each second from the Earth's surface and are lifted up into the atmosphere against the force of gravity to feed the hydrological cycle; and photosynthesis allows plants to grow year after year by absorbing carbon dioxide in the presence of sunlight. All these processes seem to proceed against the law of increase of entropy, and all occur as a result of the high quality (that is, low entropy) of the incoming solar radiation and its systematic variation with latitude. It is, of course, the solar energy that heats the Earth's surface and the atmosphere unevenly, thereby generating the global wind sys- 


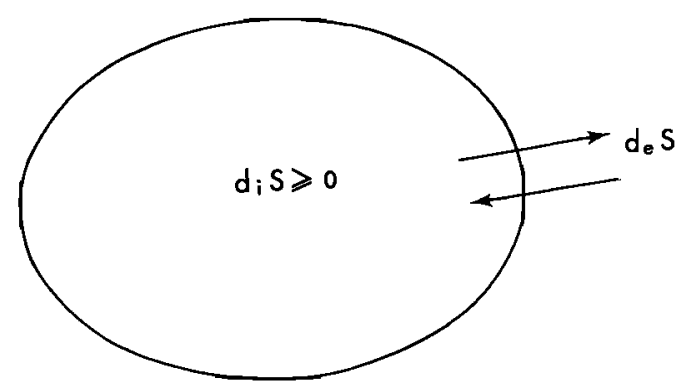

Fig. 1. Schematic diagram showing the transfer of entropy across the boundaries and the production of entropy inside a system.

tems, producing the evaporation of water as one of the vital components of the hydrological cycle, and maintaining photosynthesis, among many other processes.

We can also compare the quality of the shortwave solar radiation $\left(\lambda_{\max } \approx 0.47 \mu \mathrm{m}\right)$ with the quality of the longwave radiation $\left(\lambda_{\max } \approx 10 \mu \mathrm{m}\right)$ emitted by the Earth. According to the Planck equation the energy $\varepsilon$ of a photon is given by $\varepsilon=$ $h \nu$, where $h$ is the Planck constant and $\nu$ is the frequency, and the energy is thus inversely proportional to the wavelength of the radiation. The same amount of energy contains fewer photons in the form of solar radiation than in the form of terrestrial radiation, or in other words, the solar radiation is more organized (has higher quality) than its terrestrial counterpart. For the Earth as a whole the amount of entropy associated with the incoming solar radiation is much lower than the amount of entropy associated with the emitted terrestrial radiation, so that the climate system receives high-quality energy and returns low-quality energy to space. Thus solar radiation revitalizes the meteorological phenomena, feeds the hydrological cycle, and renovates the biosphere. If the Earth were an isolated system, there would be an unavoidable increase in entropy leading to a deathlike uniformity of the planet Earth. It is this capacity for permanent renovation that makes all natural phenomena possible in the climatic system.

Most quantities in the physical world can increase or decrease with time, but entropy must always increase. The entropy can decrease locally during a given time interval but only at the expense of a larger increase of entropy in the environment, so that it results in a net increase in the global entropy. Entropy is the "time's arrow," in the words of Eddington. It gives the direction in which time flows, but it does not provide the rate at which time is increasing, so it cannot be used as a clock. Entropy sometimes increases more rapidly and at other times more slowly; only rarely does it remain constant. The second law does not provide the speed of the degradation of energy. This degradation can be accelerated (e.g., by animal life) or slowed down (e.g., by green plants [Prigogine, 1980]).

Several papers dealing with the entropy budget of the atmosphere have been published before. Among them we can mention Wulf and Davis [1952], Lettau [1954], Dutton and Johnson [1967], Dutton [1973, 1976], Paltridge [1975], Livezey and Dutton [1976], Fortak [1979], Essex [1984], Johnson [1989], Callies [1989], and Lesins [1990].

\section{BALANCE of EnTropy}

\subsection{General Considerations}

Although the atmosphere is not in equilibrium, we will assume that there exists a state of local equilibrium, so that we can still accept the formal relations developed in equilibrium thermodynamics [Prigogine, 1962].

One of the main forms of energy in the climate system is the radiant energy. We know that the definition of entropy for a radiating system is well established when it is assumed that the system acts as a blackbody in radiative equilibrium. However, for nonblackbody radiation there is, as yet, no adequate definition of entropy (see discussions by Essex [1984], Callies [1989], and Lesins [1990]). Nevertheless, we follow the usual approach in evaluating the entropy of radiation beams by accepting the idea that the entropy fluxes are associated with the corresponding fluxes of energy. The rate of entropy carried by a radiation beam is then obtained by dividing the flux of radiative energy by the emission temperature.

The rate of change, $d s / d t$, of entropy per unit mass is given by

$$
d s / d t=Q / T,
$$

where $Q$ is the rate of diabatic heating per unit mass and $T$ is the absolute temperature. For the atmosphere the diabatic heating includes radiative heating and cooling, the release of latent heat by the phase transitions, the heating by conduction of sensible heat, and the heating by frictional dissipation:

$Q=-\frac{1}{\rho}\left\{\operatorname{div} \mathbf{F}_{\mathrm{rad}}+\rho L(e-c)+\operatorname{div} \mathbf{J}_{H}+\boldsymbol{\tau}: \operatorname{grad} \mathbf{c}\right\}$

where $\rho$ is the density of air, $L$ is the latent heat of condensation, $c$ and $e$ are the rates of condensation and evaporation per unit mass, $\mathbf{F}_{\text {rad }}$ is the net radiative flux (solar plus terrestrial), $\mathbf{J}_{H}$ is the sensible heat flux due to conduction, $\boldsymbol{\tau}$ is the wind stress tensor, and $\mathrm{c}$ is the wind velocity. The rate of diabatic heating can be separated into two components:

$$
Q=Q_{h}+Q_{f}
$$

where $Q_{f}$ indicates the frictional part of the diabatic heating, that is, the last term on the right side of (4), and $Q_{h}$ contains the remaining terms.

In the long run, for the total atmosphere the entropy remains constant, so that using (3) and (5) we have

$$
\int_{v}\left(\rho \frac{Q_{h}+Q_{f}}{T}\right) d V=0
$$

or, in terms of pressure, using the Poisson equation,

$$
\int_{v}\left(\rho \frac{Q_{h}+Q_{f}}{p^{\kappa}}\right) d V=0
$$

where the overbar indicates a long-term average (e.g., a year), $p$ is the pressure, $\kappa=R / c_{p}, R$ is the gas constant for dry air, and $c_{p}$ is the specific heat of air at constant pressure. Since $T, p$, and $Q_{f}$ are always positive, the integrals 


$$
\int_{\nu} \rho \overline{\frac{Q_{f}}{T}} d V \quad \int_{v} \overline{\rho \frac{Q_{f}}{p^{\kappa}}} d V
$$

are also always positive. This leads to the conclusion that

$$
\begin{aligned}
& \int_{\nu} \overline{\rho \frac{Q_{h}}{T}} d V<0 \\
& \int_{\nu} \rho \overline{\frac{Q_{h}}{p^{\kappa}}} d V<0 .
\end{aligned}
$$

Therefore the $Q_{h}$ and $T$ fields (or the $Q_{h}$ and $p$ fields) must be positively correlated in the atmosphere; that is, high values of $Q_{h}$ must occur at high temperatures (or at high pressures) and vice versa in order to generate entropy. This is what is actually observed in the atmosphere with heating occurring in low latitudes and at low levels, where the temperatures are higher, and cooling in high latitudes and at high levels, where the temperatures are lower. In other words, the general circulation can be maintained against the disordering effects of friction only because regions that are already relatively warm are heated whereas the relatively cold regions lose energy. The same result is obtained for the generation of available potential energy [Oort and Peixoto, 1974; Dutton, 1976].

\subsection{Balance Equation of Entropy}

The local balance equation of entropy can be obtained by substituting the expression for diabatic heating (4) into (3):

$\rho \frac{d s}{d t}=-\frac{\operatorname{div} \mathbf{F}_{\mathrm{rad}}}{T}-\frac{L \rho(e-c)}{T}-\frac{\operatorname{div} \mathbf{J}_{H}}{T}-\frac{\tau: \operatorname{grad} \mathbf{c}}{T}$

Or

$$
\begin{gathered}
\rho \frac{d s}{d t}=-\operatorname{div} \frac{1}{T}\left(\mathbf{F}_{\mathrm{rad}}+\mathbf{J}_{H}\right)-\frac{1}{T^{2}}\left(\mathbf{F}_{\mathrm{rad}}+\mathbf{J}_{H}\right) \cdot \operatorname{grad} T \\
-\frac{L \rho(e-c)}{T}-\frac{\tau: \operatorname{grad} \mathbf{c}}{T} .
\end{gathered}
$$

If the total derivatives on the left side of (10) and (11) are expanded and if we integrate the resulting equations over the entire atmosphere, we obtain

$$
\begin{gathered}
\frac{\partial}{\partial t} \int_{v} \rho s d V=-\int_{A} \rho s \mathbf{c} \cdot \mathbf{n} d A-\int_{v} \frac{1}{T}\left\{\operatorname{div} \mathbf{F}_{\mathrm{rad}}+\operatorname{div} \mathbf{J}_{H}\right. \\
+L \rho(e-c)+\boldsymbol{\tau}: \operatorname{grad} \mathbf{c}\} d V \\
\frac{\partial}{\partial t} \int_{v} \rho s d V=-\int_{A} \rho s \mathbf{c} \cdot \mathbf{n} d A-\int_{A} \frac{1}{T} \mathscr{F} \cdot \mathbf{n} d A \\
-\int_{v} \frac{1}{T^{2}} \mathscr{F} \cdot \operatorname{grad} T d V-\int_{V} \frac{L}{T} \rho(e \\
-c) d V-\int_{V} \frac{1}{T} \tau: \operatorname{grad} \mathbf{c} d V
\end{gathered}
$$

respectively, where $\mathscr{F}=\mathbf{F}_{\text {rad }}+\mathbf{J}_{H}$, and $\mathbf{n}$ is the unit vector directed outward at right angles to the boundaries of the atmosphere. The first two terms on the right side of (13) give the fluxes of entropy across the boundaries associated with the transport of mass and with the transfer of energy, respectively. For the entire atmosphere the first term on the right side of (12) and (13) vanishes at the top of the atmosphere since $\rho$ decreases exponentially with height and $s$ increases linearly; this term also vanishes at the Earth's surface where the normal component of the velocity $\mathbf{c} \cdot \mathbf{n}$ equals zero. The third and fifth terms on the right side of (13) have a bilinear form in which one of the components of the product is a flux of a given quantity and the other is the gradient of the conjugate intensive state variable [de Groot and Mazur, 1984]. For the climatic system these generation terms consist of the products of the rates at which the various irreversible processes take place (radiation, heat flow, diffusion, etc.) and the corresponding generalized force, e.g., the gradient of temperature. Equivalent versions of the preceding equations are given by Batchelor [1967], Dutton [1976], and de Groot and Mazur [1984].

Equations (12) and (13) are only approximate equations since they do not take into proper account the entropy of the radiant energy fields. This constitutes a difficult problem to deal with outside equilibrium conditions because the nonequilibrium radiation entropy is not related in a simple way to the blackbody radiation entropy. However, some attempts have been made recently to evaluate the radiation entropy [see Essex, 1984; Callies, 1989; Lesins, 1990].

Equation (13) is equivalent to Prigogine's [1962] formulation (2) for the rate of change of entropy when applied to the atmosphere as an open system. Thus the sum of the first two terms on the right side of (13) corresponds to the term $d_{e} S / d t$ in (2), while the sum of the last three terms on the right side of (13) corresponds to the term $d_{i} S / d t$ in (2). The last sum must always be nonnegative $\left(d_{t} S / d t \geq 0\right)$ since it is the only term that remains provided that we consider the atmosphere, as a whole, as an isolated system. However, the individual generation terms may not be positive everywhere, as possibly occurs in the case of radiation and in some phase transitions, such as evaporation, since the integrand may be locally negative. Nevertheless, the latent heat release, frictional dissipation, and heat diffusion terms are always positive [Batchelor, 1976]. This is easy to prove in the case of the heat diffusion term by assuming a type of Fickian law for $\mathbf{J}_{H}$,

$$
\mathbf{J}_{H}=-k \operatorname{grad} T,
$$

where $k$ is the thermal diffusivity which is positive. The contribution of the heat diffusion terms to the entropy generation is then always positive since

$$
-\int_{v} \frac{1}{T^{2}} \mathbf{J}_{H} \cdot \operatorname{grad} T d V=\int_{v} \frac{k}{T^{2}}(\operatorname{grad} T)^{2} d V>0 .
$$

\section{Observed Entropy Budget of the Atmosphere}

\subsection{Evaluation of the Balance Equation}

To evaluate the various boundary terms in (13), it is convenient to use the mean value theorem and to introduce an appropriate reference or equivalent temperature $T^{*}$ for each component so that 


$$
\frac{1}{T^{*}} \equiv\left(\int \frac{1}{T} \mathscr{F}_{F} \cdot \mathbf{n} d A\right)\left(\int \mathscr{F}_{P} \cdot \mathbf{n} d A\right)^{-1} .
$$

The mean value theorem can be used since in the atmosphere the integrand functions (fluxes) are predominantly of the same sign. For the radiation term in (13) we find

$$
\int-\frac{1}{T} \mathbf{F}_{\mathrm{rad}} \cdot \mathbf{n} d A=\frac{-1}{T_{\mathrm{rad}}^{*}} \int \mathbf{F}_{\mathrm{rad}} \cdot \mathbf{n} d A=G_{\mathrm{rad}} / T_{\mathrm{rad}}^{*},
$$

where $G_{\mathrm{rad}}$ is the net radiative flux across the boundaries into the atmosphere (since $n$ is directed outward) and $T_{\text {rad }}^{*}$ is the reference radiative temperature. We can obtain similar expressions for the other boundary terms. Thus (13) can be rewritten symbolically in the form

$$
\frac{\partial S}{\partial t}=\frac{G_{\mathrm{rad}}}{T_{\mathrm{rad}}^{*}}+\frac{G_{\mathrm{SH}}}{T_{\mathrm{SH}}^{*}}+\sigma_{\mathrm{rad}}+\sigma_{\mathrm{LH}}+\sigma_{\mathrm{SH}}+\sigma_{\mathrm{dis}} .
$$

In this equation, $S=\int_{v} \rho s d v$ is the total entropy of the atmosphere, and $\sigma_{\mathrm{rad}}, \sigma_{\mathrm{LH}}, \sigma_{\mathrm{SH}}$, and $\sigma_{\mathrm{dis}}$ denote the rates of generation of entropy by radiation, release of latent heat, heat conduction, and dissipation of kinetic energy, respectively, which are all associated with irreversible processes in the atmosphere.

For the atmosphere as a whole we will assume that the net rate at which latent heat is released can be approximated by the product of the observed precipitation rate at the Earth's surface and the latent heat of condensation:

$$
-L \int_{0}^{\infty} \rho(e-c) d z \approx \rho_{w} L P
$$

where $P$ is the precipitation rate and $\rho_{w}$ is the density of water. A large generation of entropy $\sigma_{\mathrm{LH}}$ must be connected with this phase transition. The evaporation rate at the Earth's surface is not included in this term since we only consider here the entropy budget of the atmosphere itself. The mixing of tropical and polar air masses (with different temperatures and humidities) also contributes to the generation of entropy through the diffusion terms in (16).

For a sufficiently long interval of time (e.g., a year) we may assume that on the average the atmosphere is in a steady state. Therefore, averaging of the global entropy balance equation (16) over time leads to

$\left(\overline{\frac{G_{\mathrm{rad}}}{T_{\mathrm{rad}}^{*}}}\right)+\left(\overline{\frac{G_{\mathrm{SH}}}{T_{\text {SH }}^{*}}}\right)+\bar{\sigma}_{\mathrm{rad}}+\bar{\sigma}_{\mathrm{LH}}+\bar{\sigma}_{\mathrm{SH}}+\bar{\sigma}_{\mathrm{dis}}=0$.

It is worth noting that under steady state conditions the corresponding energy terms must also obey the energy balance equation

$$
\bar{G}_{\mathrm{rad}}+\bar{G}_{\mathrm{LH}}+\bar{G}_{\mathrm{SH}}=0
$$

where

$$
G_{\mathrm{LH}}=-\int \mathbf{F}_{\mathrm{LH}} \cdot \mathbf{n} d A
$$

represents the latent heat term and $\mathbf{F}_{\mathrm{LH}}$ is the flux of latent heat.
Let us discuss the reference temperature further. This temperature represents a measure of the average temperature at which the processes occur. If we assume that the relative fluctuations of the reference temperatures and of $G$ are small, that is, $T^{\prime} / T^{*} \ll 1$ and $G^{\prime} / G \ll 1$, then

$$
\overline{G / T^{*}}=\bar{G} / \overline{T^{*}}-\overline{G^{\prime} T^{\prime}} / \bar{T}^{* 2},
$$

where $T^{\prime} \equiv T^{*}-\bar{T}^{*}$ and $\bar{T}^{*}$ is the time average of the instantaneous reference temperature and not the reference temperature of the mean state, and similarly for $G^{\prime}$. The first term on the right side of (19) represents the contribution of the time mean flux of entropy, and the second term represents the transient eddy contribution. Since $\bar{T}^{*}$ is very large in comparison to the fluctuations $T^{\prime}$, the last term in (19) can be neglected. Further, as our preliminary calculations of $\bar{T}^{*}$ have shown, we can use the horizontally averaged temperature, $\bar{T}$, instead of $\bar{T}^{*}$. Thus (17) can be written as

$$
\bar{G}_{\mathrm{rad}} / \bar{T}_{\mathrm{rad}}+\bar{G}_{\mathrm{SH}} / \bar{T}_{\mathrm{SH}}+\bar{\sigma}_{\mathrm{rad}}+\bar{\sigma}_{\mathrm{LH}}+\bar{\sigma}_{\mathrm{SH}}+\bar{\sigma}_{\mathrm{dis}}=\mathbf{0},
$$

which is used in the following calculations.

\subsection{Global Entropy Budget}

To evaluate the entropy budget of the atmosphere, we begin by estimating the first two terms in (20) that represent the fluxes of entropy across the top and bottom boundaries of the atmosphere. As shown by (14) and (15), this requires computing both the mean energy fluxes $G$ across the boundaries and the corresponding (energy flux weighted) reference temperatures $T^{*}$ for these boundaries. To do the calculations properly, we would need to know the global distributions of both the temperature and radiation fluxes at the top of the atmosphere as well as global distributions of the temperature, radiation, and sensible heat fluxes at the Earth's surface. Of all these quantities the temperature distributions are well known. Also, at the top of the atmosphere the radiation fields are reasonably well known from satellite observations, but at the surface the flux fields are relatively poorly known.

The mean energy flux values at the top and bottom of the atmosphere, as given by Peixoto and Oort [1984, Figure 7] in the form of percentages of the total incoming solar radiation, are used to estimate the values of the generation terms, assuming that the annual mean incident solar radiation is $\mathbf{3 4 0}$ $\mathrm{W} \mathrm{m} \mathrm{m}^{-2}$. The corresponding reference temperatures could be estimated by taking into account the characteristic global distributions of the different fluxes and temperature fields, but as we mentioned earlier, we will use the averaged temperature instead. The results are presented in Figure 2, which gives the chosen values for the mean temperatures, the energy fluxes, and the corresponding entropy fluxes at the top and bottom of the atmosphere. The mean temperature for the downward solar radiation flux and the upward fluxes at the Earth's surface were assumed to be $5760 \mathrm{~K}$ (the effective temperature of the Sun) and $288 \mathrm{~K}$ (the mean temperature at the Earth's surface), respectively. We will use a positive sign for the flux of entropy when it represents a gain of entropy for the atmosphere and a negative sign in case of a loss of entropy for the atmosphere.

The three terms in the upward flux of entropy at the top of 
TERRESTRIAL RADIATION

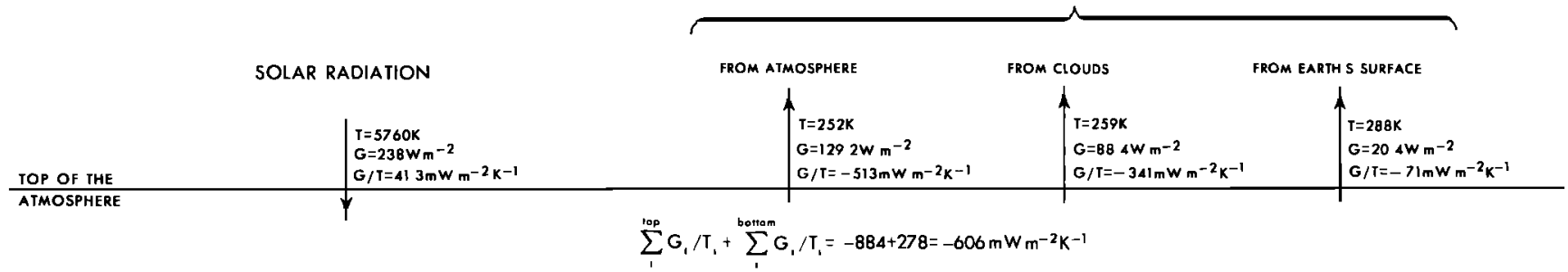

ENTROPY GENERATION PROCESSES

ABSORPTION SOLAR
RADIATION
$T=252 \mathrm{~K}$
$\rho Q=68 \mathrm{~W} \mathrm{~m}^{-2}$
$\sigma=258 \mathrm{~m} \mathrm{~W} \mathrm{~m}^{-2} \mathrm{~K}^{-1}$

\begin{tabular}{l} 
ABSORPTION TERRESTRIAL \\
RADIATION \\
$T=252 \mathrm{~K}$ \\
$\rho Q=476 \mathrm{~W} \mathrm{~m} \mathrm{~m}^{-2}$ \\
$\sigma=24 \mathrm{~mW} \mathrm{~m}^{-2 \mathrm{~K}-1}$ \\
\hline
\end{tabular}

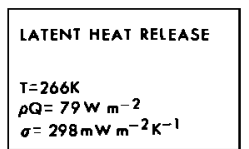

$\sum \sigma_{1}=589 \mathrm{mWm}^{-2} \mathrm{~K}^{-1}$
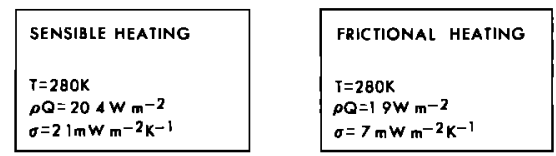

$\sigma=21 m W_{m^{-2}} K^{-1}$

$\sigma=7 \mathrm{~mW} \mathrm{~m}^{-2} \mathrm{~K}^{-1}$

\begin{tabular}{|c|c|c|c|}
\hline EARTH'S & SOLAR RADIATION & $\begin{array}{c}\text { SENSIBLE HEAT } \\
\text { a }\end{array}$ & TERRESTRIAL RADIATION \\
\hline SURFACE & $\begin{array}{l}T=5760 \mathrm{~K} \\
G=170 \mathrm{~W} \mathrm{~m}^{-2} \\
G / \mathrm{T}=-295 \mathrm{~m} \mathrm{~W} \mathrm{~m}^{-2} \mathrm{~K}^{-1}\end{array}$ & $\begin{array}{l}T=288 K \\
G=204 \mathrm{Wm}^{-2} \\
G / t=71 \mathrm{~mW} \mathrm{~m}^{-2} \mathrm{~K}^{-1}\end{array}$ & $\begin{array}{l}T=288 K \\
G=68 \mathrm{~W} \mathrm{~m}^{-2} \\
G / T=236 \mathrm{~m} \mathrm{~W} \mathrm{~m}^{-2} \mathrm{~K}^{-1}\end{array}$ \\
\hline
\end{tabular}

Fig. 2. Entropy budget of the global atmosphere estimated for annual mean conditions. Shown are the equivalent temperature $\mathrm{T}^{*}$ in $\mathrm{K}$, the energy flux $G$ in W m $\mathrm{m}^{-2}$, and the entropy flux $G / T^{*}$ in units of $\mathrm{mW} \mathrm{m} \mathrm{m}^{-2} \mathrm{~K}^{-1}$ for each energy component at the top of the atmosphere and at the Earth's surface. A positive (negative) sign is used when the atmosphere gains (loses) entropy. The boxes in the middle of the figure contain estimates for each component of the atmospheric temperature where the absorption takes place in $\mathrm{K}$, the rate at which energy is absorbed, $\rho Q$, in $\mathrm{W} \mathrm{m}{ }^{-2}$, and the rate of entropy production $\sigma$ in units of $\mathrm{mW} \mathrm{m}^{-2} \mathrm{~K}^{-1}$.

the atmosphere are associated with the fluxes of infrared radiation emitted by the Earth's surface (through the spectral window 8.5-11.0 $\mu \mathrm{m}$ ), the atmosphere, and the clouds. For the emission of infrared radiation by the atmosphere into space we used a mean temperature of $252 \mathrm{~K}$, and for the emission by the clouds we used a value of $259 \mathrm{~K}$ which corresponds to the mean temperature of the cloud tops according to Newell et al. [1974].

At the bottom of the atmosphere we estimated the values of fluxes of entropy associated with the incident solar radiation $\left(-29.5 \mathrm{~mW} \mathrm{~m}^{-2} \mathrm{~K}^{-1}\right)$, the upward sensible heat flux $\left(71 \mathrm{~mW} \mathrm{~m}^{-2} \mathrm{~K}^{-1}\right)$, and the emitted longwave radiation $\left(236 \mathrm{~mW} \mathrm{~m}^{-2} \mathrm{~K}^{-1}\right)$. As expected from the high quality of the incoming solar radiation, the fluxes of entropy in the solar radiation are small both at the top and at the bottom of the atmosphere with values of 41.3 and $-29.5 \mathrm{~mW} \mathrm{~m}^{-2} \mathrm{~K}^{-1}$, respectively.

When we add all boundary fluxes together, the total flux of entropy at the top of the atmosphere is $-884 \mathrm{~mW} \mathrm{~m}^{-2} \mathrm{~K}^{-1}$, and the total flux at the bottom of the atmosphere is $278 \mathrm{~mW}$ $\mathrm{m}^{-2} \mathrm{~K}^{-1}$. Thus according to (17) the mean rate of generation of entropy by internal processes in the atmosphere under steady conditions must be $\sigma=884-278=606 \mathrm{~mW} \mathrm{~m}^{-2}$ $\mathbf{K}^{-1}$.

As we see from the values given in Figure 2, the total amount of entropy exported by the climate system to space is $-71-513-341=-925 \mathrm{~mW} \mathrm{~m} \mathrm{~m}^{-2} \mathrm{~K}^{-1}$. This value is about 22 times the amount of entropy imported by the incoming solar radiation at the top of the atmosphere (41.3 $\mathrm{mW} \mathrm{m}^{-2} \mathrm{~K}^{-1}$ ).

The largest fluxes of entropy are associated with the longwave radiation fluxes $\left(236 \mathrm{~mW} \mathrm{~m}^{-2} \mathrm{~K}^{-1}\right.$ at the Earth's surface and $-925 \mathrm{~mW} \mathrm{~m} \mathrm{~m}^{-2} \mathrm{~K}^{-1}$ at the top). The flux of entropy associated with turbulent and molecular diffusion processes is much smaller $\left(71 \mathrm{~mW} \mathrm{~m}^{-2} \mathrm{~K}^{-1}\right)$.

It is important that the rate of generation of entropy within the atmosphere also be evaluated in an independent way. Thus we will try to compute the rate of production of entropy within the atmosphere directly from the individual contributions by the various irreversible processes that occur inside the atmosphere. We again make use of the energy flux values of Peixoto and Oort [1984]. Let us consider as an example the evaluation of the generation of entropy due to the absorption of solar radiation. This term can be evaluated assuming that the absorption occurs near the 500 -mbar level where the temperature is about $252 \mathrm{~K}$, so that

$$
\sigma_{\mathrm{rad}}^{\mathrm{SW}}=\frac{68}{252}-\frac{68}{5760}=258 \mathrm{~mW} \mathrm{~m}^{-2} \mathrm{~K}^{-1}
$$

For the latent heat release we assume a net precipitation rate of $1 \mathrm{~m} \mathrm{yr}^{-1}$ and a condensation temperature of $266 \mathrm{~K}$, so that

$$
\sigma_{\mathrm{LH}}=\frac{\rho_{w} L P}{T}=\frac{79}{266}=298 \mathrm{~mW} \mathrm{~m}^{-2} \mathrm{~K}^{-1},
$$

which represents the largest entropy generation term.

For the sensible heat exchange processes we assumed an average temperature of $280 \mathrm{~K}$ as representative of the bottom layer of the atmosphere. The estimated temperatures, the rate of diabatic heating, and the rate of entropy generation for the individual processes are shown in the rectangular boxes in the middle of Figure 2. The values of the entropy generation rates by the various processes are also presented in Table 1 . When we add all these contribu- 
TABLE 1. Entropy Generation by the Various Processes Occurring Inside the Global Atmosphere

\begin{tabular}{lc}
\hline & $\begin{array}{c}\text { Entropy Generation } \\
\text { Values, } \mathrm{mW} \mathrm{m} \mathrm{m}^{-2} \mathrm{~K}^{-1}\end{array}$ \\
\hline Absorption of solar radiation & $\sigma_{\mathrm{rad}}^{\mathrm{SW}}=258$ \\
Absorption of terrestrial radiation & $\sigma_{\mathrm{rad}}^{\mathrm{LW}}=24$ \\
Release of latent heat & $\sigma_{\mathrm{LH}}=298$ \\
Sensible heat flux & $\sigma_{\mathrm{SH}}=2$ \\
Turbulent and molecular diffusion of heat & $\sigma_{\mathrm{dis}}=7$ \\
Total generation of entropy & $\sigma_{\mathrm{dis}}=589$ \\
\hline
\end{tabular}

tions in Table 1 together, the total rate of entropy generation ( $589 \mathrm{~mW} \mathrm{~m}^{-2} \mathrm{~K}^{-1}$ ) can be compared with the previous value obtained from the global budget of the atmospheric energy $\left(606 \mathrm{~mW} \mathrm{~m}^{-2} \mathrm{~K}^{-1}\right)$. The difference is only $17 \mathrm{~mW} \mathrm{~m}{ }^{-2} \mathrm{~K}^{-1}$ and can be attributed to (apart from errors) the approximations made and to some neglected effects (e.g., mixing of air masses).

In summary, the values presented in Figure 2 and Table 1 show that the absorption of solar radiation and the release of latent heat are by far the largest sources of entropy in the atmosphere. The rate of production of entropy associated with the absorption of longwave radiation is an order of magnitude smaller than that due to solar radiation since the temperatures of the infrared emission and absorption do not differ substantially. Among the nonradiative processes the water phase transitions dominate the entropy generation, whereas the rates of generation due to the sensible heat fluxes and friction are found to be relatively small.

The relevant temperatures we have chosen for the various generating processes involve some degree of arbitrariness. In order to analyze the influence of this choice we present in Table 2 some estimates of the entropy-generating terms for various temperatures. As can be seen, the most sensitive process in terms of relative change is the one related with the absorption of longwave radiation. Those related with the other processes are much smaller. In summary, the fluctuations of $\sigma$ are relatively small, so that our main conclusions would not be affected.

\subsection{Regional Entropy Budgets}

To obtain information about the distribution of the entropy sources and sinks in the atmosphere, we present some results of the budgets on a regional scale. Because of the sphericity of the Earth and the nonhomogeneous distribution of water and land there are large differences in solar insolation with respect to both latitude and longitude. However, the differences in the solar heating are, of course, most pronounced in the latitudinal direction and in particular

TABLE 2. Influence of Temperature on the Estimates of the Generation Processes for the Global Atmosphere

\begin{tabular}{cccccc}
\hline$T_{\text {rad }}^{\text {SW }}$ & $\sigma_{\text {rad }}^{\text {SW }}$ & $T_{\text {rad }}^{\text {LW }}$ & $\sigma_{\text {rad }}^{\text {LW }}$ & $T_{\text {LH }}$ & $\sigma_{\mathrm{LH}}$ \\
\hline 248 & 262 & 248 & 26.7 & 262 & 302 \\
250 & 260 & 250 & 25.1 & 264 & 300 \\
252 & 258 & 252 & 23.6 & 266 & 298 \\
254 & 256 & 254 & 22.1 & 268 & 296 \\
256 & 254 & 256 & 20.7 & 270 & 293 \\
\hline
\end{tabular}

The temperatures are in $\mathrm{K}$, and the $\sigma$ are in $\mathrm{mW} \mathrm{m}^{-2} \mathrm{~K}^{-1}$.
TABLE 3. Fluxes of Entropy Across the Boundaries of the Globe and the Equatorial, $15^{\circ}-70^{\circ} \mathrm{N}$, and Polar Regions

\begin{tabular}{lcccc}
\hline & Lateral & Top & Bottom & Sum \\
\hline Globe & $\ldots$ & -884 & 278 & -606 \\
Equatorial & -161 & -925 & 257 & -829 \\
$15^{\circ}-70^{\circ} \mathrm{N}$ & 32 & -851 & 289 & -530 \\
Polar & 331 & -747 & 250 & -166 \\
\hline
\end{tabular}

Units are $\mathrm{mW} \mathrm{m} \mathrm{m}^{-2} \mathrm{~K}^{-1}$

between the equatorial zone and the poles. Thus two limited regions were chosen for further study: an equatorial region bounded by the latitudinal walls of $15^{\circ} \mathrm{N}$ and $15^{\circ} \mathrm{S}$ and a polar cap northward of $70^{\circ} \mathrm{N}$. Conceptually, the only difference between these regional budgets and the global budget comes from the existence of lateral boundaries. Thus we must also take into account the advective entropy fluxes associated with the transports of potential energy and sensible heat across the latitudinal walls. For reasons of completeness we will also present the results for the intermediate $15^{\circ}-70^{\circ} \mathrm{N}$ region where the gradients in most meteorological quantities are very large and averages are less well defined.

Our basic estimates of the solar and terrestrial radiative fluxes at the top of the atmosphere are for each region based on the satellite data presented by Oort and Peixoto [1983, Figure 19]. For the atmospheric absorption of solar radiation we used everywhere a value of $20 \%$ of the solar radiation received at the top of the atmosphere. In the longwave radiation calculations we assumed that $29 \%$ of the total outgoing longwave radiation emitted at the top of the atmosphere is emitted by the Earth's surface and that the remaining $71 \%$ comes from longwave radiation absorbed in the atmosphere. For the surface flux of sensible heat we used Budyko's [1986, Figure 2.9] results as a guide. Finally, for the release of latent heat we assumed mean precipitation rates of $150 \mathrm{~cm} \mathrm{yr}^{-1}$ in the equatorial region, $80 \mathrm{~cm} \mathrm{yr}^{-1}$ in the $15^{\circ}-70^{\circ} \mathrm{N}$ belt, and $20 \mathrm{~cm} \mathrm{yr}^{-1}$ in the north polar region [after Jaeger, 1976].

Our estimates of the horizontal energy fluxes across the latitudinal walls are based on the rawinsonde data by Oort and Rasmusson [1971, Tables C2 and C3]. In these computations we disregarded the horizontal radiative and turbulent fluxes of heat because they are very small. For the temperatures associated with the various energy fluxes we used again the data from Oort and Rasmusson [1971, Table A4]. We assumed that the absorption of shortwave solar radiation and the emission of longwave terrestrial radiation both take place just above the 500-mbar level and that the latent heat release occurs near the 500 -mbar level in the equatorial region, near 700 mbar at mid-latitudes, and near 850 mbar at polar latitudes.

The results for the various fluxes are presented in Table 3. These show a lateral export of entropy away from the equatorial belt $\left(-161 \mathrm{~mW} \mathrm{~m}^{-2} \mathrm{~K}^{-1}\right)$. On the other hand, there is a strong lateral inflow of entropy into the polar region $\left(331 \mathrm{~mW} \mathrm{~m}^{-2} \mathrm{~K}^{-1}\right)$. The values at the top show that the export of entropy to space is larger in the equatorial atmosphere than in the polar atmosphere.

The sums of the boundary terms presented in the last column of Table 3 give a rough estimate of the net rate of generation of entropy inside each region. However, in view of their importance the individual rates of entropy generation 
TABLE 4. Entropy Source Processes for the Globe and the Equatorial, $15^{\circ}-70^{\circ} \mathrm{N}$, and Polar Regions

\begin{tabular}{lcccccc}
\hline & $\begin{array}{c}\text { Absorption } \\
\text { of Solar } \\
\text { Radiation }\end{array}$ & $\begin{array}{c}\text { Absorption of } \\
\text { Terrestrial } \\
\text { Radiation }\end{array}$ & $\begin{array}{c}\text { Latent } \\
\text { Heat } \\
\text { Release }\end{array}$ & $\begin{array}{c}\text { Sensible } \\
\text { Heating }\end{array}$ & $\begin{array}{c}\text { Frictional } \\
\text { Heating }\end{array}$ & Sum \\
\hline Globe & 258 & 24 & 298 & 2 & 7 & 589 \\
Equatorial & 335 & 25 & 446 & 2 & 7 & 815 \\
$15^{\circ}-70^{\circ} \mathrm{N}$ & 253 & 20 & 231 & 3 & 7 & 514 \\
Polar & 56 & 18 & 61 & 5 & 7 & 147 \\
\hline
\end{tabular}

Units are $\mathrm{mW} \mathrm{m}^{-2} \mathrm{~K}^{-1}$.

by the various generating processes were also estimated directly for each region, as discussed for the globe in section 3.2. The results are given in Table 4. The regional friction effects are not well known. However, we have assumed here that the regional values are the same as the global value of 7 $\mathrm{mW} \mathrm{m}^{-2} \mathrm{~K}^{-1}$. As expected, the main generation processes in the equatorial region are related to the release of latent heat and the absorption of solar radiation. The generation due to the absorption of longwave terrestrial radiation and that due to sensible and latent heat are estimated to be relatively small. In the polar region the contributions of latent heat release and absorption of solar radiation are found to be about equally important. As expected, the estimated values of the entropy flux and production terms for the $15^{\circ}-70^{\circ} \mathrm{N}$ region lie in between the estimates for the equatorial and polar regions and are close to the global estimates. The total values in the last column of Tables 3 and 4 show clearly that the equatorial region is by far more active than the polar region as far as the entropy generation is concerned.

The differences in the total rates of entropy generation as computed indirectly in Table 3 and directly in Table 4 are relatively small. They can be attributed to the various approximations in the formulation of the problem and in the actual calculations.

\section{Concluding Remarks}

In the long-term mean the outgoing flux of longwave radiation to space equals the incoming flux of solar energy almost exactly, but the corresponding entropy fluxes are quite different. In fact, the entropy exported by the outgoing longwave radiation is found to be about 22 times larger than the entropy associated with the incoming solar radiation. The net export of entropy to space by the climate system must be equal to the internal production of entropy by the irreversible processes that occur within the system.

The computation of the entropy fluxes at the boundaries depends critically on the temperatures chosen for the surface of the globe, the various layers and regions of the atmosphere, and the clouds.

The most important processes for the generation of entropy involve the release of latent heat and the radiative interactions, particularly those associated with the absorption of solar radiation. The contributions to the entropy balance associated with the sensible heat fluxes and with frictional dissipation are much smaller.

As might be expected, the entropy sources and sinks inside the climate system are not uniformly distributed because of the sphericity of the Earth and the nonuniformity of the Earth's land and ocean surfaces. In the atmosphere the generation of entropy is much larger in low latitudes than in middle and high latitudes, mainly because of the greater release of latent heat and the stronger absorption of solar radiation.

The study of the entropy budgets for selected atmospheric regions provides preliminary information on the spatial distribution of the main entropy sources and sinks. However, there is much room for improvement: (1) A more accurate and detailed knowledge of the components of the diabatic heating and of the surface fluxes, together with their temporal and spatial distributions, is needed. (2) The evaluation of the role of oceanic processes in producing entropy should be attempted. (3) A more detailed level-by-level analysis should be performed on a monthly basis so that the contributions by vertical and horizontal transports of entropy may be estimated and the spatial distributions of the sources and sinks of entropy can be analyzed.

In summary, the study of the entropy budget has proved to be important to reach a better understanding of the physical processes and mechanisms involved in the climate.

Acknowledgments. We would like to thank Kerry Cook, Yoshio Kurihara, Gareth Williams, and the anonymous reviewers for their constructive comments, and Joyce Kennedy for typing the various versions of the manuscript. The financial support by the National Oceanic and Atmospheric Administration under grant NA87-EAD00039 and by the National Science Foundation under grant ATM89-11293 for the visits of J. P. Peixoto to Princeton, New Jersey, and by the EEC for the visits of A. H. Oort to Lisbon, Portugal, is gratefully acknowledged.

\section{REFERENCES}

Batchelor, G. K., Introduction to Fluid Dynamics, Cambridge University Press, New York, 1967.

Budyko, M. I., The Evolution of the Biosphere, 423 pp., D. Reidel, Norwell, Mass., 1986.

Callies, U., Entropy production by atmospheric scattering of light, Contrib. Atmos. Phys., 62, 212-226, 1989.

de Groot, S. R., and P. Mazur, Non-equilibrium Thermodynamics, 510 pp., Dover, Mineola, N. Y., 1984.

Dutton, J. A., The global thermodynamics of atmospheric motion, Tellus, 25, 89-110, 1973.

Dutton, J. A., The Ceaseless Wind: An Introduction to the Theory of Atmospheric Motion, 556 pp., McGraw-Hill, New York, 1976. (Reprinted and revised, 617 pp., Dover, Mineola, N. Y., 1986).

Dutton, J. A., and D. R. Johnson, The theory of available potential energy and a variational approach to atmospheric energetics, $A d v$. Geophys., 12, 333-436, 1967.

Essex, C., Radiation and the irreversible thermodynamics of climate, J. Atmos. Sci., 41, 1985-1991, 1984.

Fortak, H. G., Entropy and climate, in Man's Impact on Climate, edited by W. Bach, J. Pankrath, and W. Kellogg, pp. 1-14, Elsevier Science, New York, 1979.

Jaeger, L., Monthly precipitation maps for the entire Earth (in German), Ber. Dtsch. Wetterdienstes, 18(139), 38 pp., 1976. 
Johnson, D. R., The forcing and maintenance of global monsoonal circulations: An isentropic analysis, Adv. Geophys., 31, 43-316, 1989.

Lesins, G. B., On the relationship between radiative entropy and temperature distributions, J. Atmos. Sci., 47, 795-803, 1990.

Lettau, H., A study of the mass, momentum and energy budget of the atmosphere, Arch. Meteorol. Geophys. Bioklimatol., 7, 133$157,1954$.

Livezey, R. E., and J. A. Dutton, The entropic energy of geophysical fluid systems, Tellus, 28, 138-158, 1976.

Newell, R. E., J. W. Kidson, D. G. Vincent, and G. J. Boer, The General Circulation of the Tropical Atmosphere, vol. 2, 371 pp. MIT Press, Cambridge, Mass., 1974.

Oort, A. H., and J. P. Peixoto, The annual cycle of the energetics of the atmosphere on a planetary scale, J. Geophys. Res., 79, 2705-2719, 1974.

Oort, A. H., and J. P. Peixoto, Global angular momentum and energy balance requirements from observations, Adv. Geophys., $25,355-490,1983$.

Oort, A. H., and E. M. Rasmusson, Atmospheric circulation statistics, NOAA Prof. Pap. 5, 323 pp., U.S. Gov. Print. Off., Washington, D. C., 1971.

Paltridge, G. W., Global dynamics and climate-A system of minimum entropy exchange, Q. J. R. Meteorol. Soc., 101, 475484, 1975.

Peixoto, J. P., and A. H. Oort, Physics of climate, Rev. Mod. Phys., $56,365-429,1984$.

Prigogine, I., Introduction to Non-equilibrium Thermodynamics, 119 pp., Wiley Interscience, New York, 1962.

Prigogine, I., From Being to Becoming, 272 pp., W. H. Freeman, New York, 1980

Wulf, $O$. R., and L. Davis, Jr., On the efficiency of the engine driving the atmospheric circulation, J. Meteorol., 9, 79-82, 1952.

M. de Almeida, European Centre for Medium-Range Weather Forecasts, Shinfield Park, Reading, Berkshire RG2 9AX, England. A. H. Oort, Geophysical Fluid Dynamics Laboratory/NOAA, Princeton University, P.O. Box 308, Princeton, NJ 08542.

J. P. Peixoto and A. Tomé, Instituto Geofisico do Infante D. Luis Faculdade de Ciencias, Rua da Escola Politécnica, 58, 1200 Lisbon, Portugal.

(Received February 20, 1990;

revised March 5, 1991;

accepted March 8, 1991.) 